\title{
Trastornos psiquiátricos frecuentes post infarto agudo al miocardio y su relación pronóstica. Revisión de la literatura
}

\author{
Manuel Mallol Simmonds ${ }^{*}$
}

Resumen: La enfermedad cardiovascular es la principal causa de muerte a nivel mundial. De ella, el Infarto Agudo al Miocardio (IAM) y el Accidente Cerebrovascular (AVC) se encuentran fuertemente asociados a los cambios ambientales y sociales del último tiempo, tales como aumento de ingesta de comida rápida, sedentarismo, tabaquismo, entre otros. Algunos trastornos psiquiátricos que aparecen a corto y largo plazo posterior al evento coronario agudo tienen una implicancia directa en la morbimortalidad cardiovascular y global. De ellos, la depresión representa el trastorno psiquiátrico más frecuente, pudiendo encontrarse en 3 de cada 10 pacientes que tuvieron un IAM. La depresión aumenta las hospitalizaciones, disminuye la adherencia terapéutica y empeora el pronóstico cardiovascular a largo plazo. La fisiopatología en lo anterior es explicada en parte por alteraciones en el eje neuroendocrino y la respuesta al estrés, mientras que otros fenómenos permanecen aún desconocidos. Dentro de otros trastornos psiquiátricos asociados a un IAM se encuentran el delirium, ansiedad y trastornos de la personalidad. En función de lo anterior, se hace necesaria la incorporación de un sistema continuo de monitoreo y apoyo en salud mental en este grupo de pacientes para mejorar tanto su pronóstico como la calidad de vida.

Palabras clave: Infarto al miocardio; Enfermedad Cardiovascular; Depresión; Trastornos psiquiátricos; Delirium; Salud y ambiente.

Abstract: Cardiovascular disease (CVD) is the main world's cause of death. Of them, Myocardial infarction (MI) and Stroke are strongly related with environment factors like unhealthy diet, sedentary lifestyle, smoking, between others. Some psychiatric disorders seem to have a direct impact in the global and cardiovascular morbimortality of these group of patients. Depression represents the most frequently one ( 3 of $10 \mathrm{Ml}$ patients have depressive symptoms), increasing hospital admissions, decreasing therapeutic adherence and getting worsen cardiovascular prognosis. Pathophysiology involved are explain due to dysregulation of neurohormonal axis, mispaired stress-response and others that remain unclear. Others psychiatric disorders associated with MI include delirium, anxiety and personality disorders. It is necessary to start new continuous mental health monitoring system in this group of patients. Many trials emphasize the need for the incorporation of depression as an important health axis to improve life quality and prognosis of cardiovascular patients.

Keywords: Myocardial Infarction; Cardiovascular Disease; Depression; Psychiatric disorders; Delirium; Environment and Health.

Fecha de envío: 9 de marzo de 2017 - Fecha de aceptación: 19 de junio de 2017

\section{Introducción}

La enfermedad cardiovascular es la principal causa de muerte a nivel mundial, con un aproximado de 12 millones de muertes por año (OMS, 2015), representando un problema sanitario mayor, requiriendo estrategias globales y sincrónicas por diferentes asociaciones de especialistas para combatir dicha incidencia, muchas de ellas sin efectos en términos de reducción significativa de muertes cardiovasculares al año. Existe una predilección hacia el sexo masculino y mayores de 60 años, más aún aquellos que siguen hábitos de vida de riesgo para el desarrollo de enfermedad cardiovascular, como son el sedentarismo, dieta rica en hidratos de carbono altamente refinados, tabaquismo, etc. En Chile, la epidemiología mundial se replica, siendo las enfermedades cardiovasculares la principal causa de muerte, dentro de las cuales predominan la cardiopatía coronaria y el Infarto Agudo del Miocardio (Lanas et al., 2008).

Un tercio de los pacientes que sufren un IAM fallecen entre las 24 horas posterior al inicio, y muchos de los sobrevivientes tienen una importante morbilidad a corto y largo plazo (Reed et al., 2016).

Dentro de la morbilidad fundamental destacan eventos arrítmicos malignos, daños estructurales fulminantes y psiquiátricos a largo plazo.

(1) Departamento de Medicina Interna Sur, Facultad de Ciencias Médicas, Escuela de Medicina. Universidad de Santiago de Chile.

*Autor de correspondencia: manuel.mallol@usach.cl 
En esta revisión se describirán los principales artículos de la literatura asociados a trastornos psiquiátricos frecuentes como agentes modificadores de la morbimortalidad de los pacientes que han sufrido un IAM, siendo un punto fundamental en el abordaje y seguimiento posterior al tratamiento médico inmediato de esta patología.

\section{Trastornos psiquiátricos post IAM}

\section{Depresión}

La depresión post IAM es el trastorno psiquiátrico más frecuente post IAM, con una relación de 4:1 respecto a las personas sanas de edad comparable (A Meijer et al., 2013). La depresión con síntomas somáticos es la que tiene la mayor asociación al riesgo elevado, no así la cognitiva (Roest et al., 2013). La prevalencia de síntomas psicóticos es baja, principalmente asociada a condiciones psiquiátricas preexistentes.

Un 27.7\% de pacientes post IAM durante su seguimiento manifestó síntomas compatibles a depresión acorde a los criterios del DSMIV, sin que ello tuviese diferencias estadísticamente significativas entre grupos etarios, estado civil, clasificación Killip, escolaridad ni estado laboral (Blumel et al., 2005)

La edad avanzada, sexo femenino, historia de cirugía de revascularización miocárdica, IAM sin elevación del segmento ST y ansiedad previa son predictores de depresión y ansiedad persistentes, las que a su vez tienen impacto en la morbimortalidad cardiovascular y global en este grupo de pacientes, debiendo ser abordadas al momento de la aproximación terapéutica inicial (Alhurani et al., 2015).

\section{IAM y depresión en Chile}

En Chile, la prevalencia de síntomas depresivos fue de 44,4\%, con una disminución significativa al control de los 3 meses en el grupo que no fue tratado con terapia de revascularización, mientras que en el grupo que sí la recibió se mantuvieron presentes (Calderón et al., 2010). Además, la presencia de factores psicosociales (depresión, ansiedad, apoyo social percibido) parece tener un rol sinérgico y negativo en el pronóstico cardiovascular.

Por otra parte, el $27.7 \%$ de los pacientes con eventos coronarios agudos mostraron depresión en algún momento de su vida, siendo la mayoría mujeres. En los pacientes hospitalizados esta cifra es mayor (38,3\%), sin embargo, dicho porcentaje difiere del énfasis terapéutico que se le da a este tipo de pacientes, el cual, sin estar calculado, se infiere ser poco acorde a la cifra antes descrita (Blumel et al., 2005).

\section{Tratamientos en la depresión y mortalidad cardiovascular}

La terapia cognitivo - conductual, conocida por ser de gran utilidad en el tratamiento de pacientes con depresión (sin patología cardíaca) debido a la capacidad de poder abordar diferentes problemas y ejes (como la conducta y el estrés), iniciada 17 días post IAM, mostró una mejoría en los outcomes psicosociales aislados a los 6 meses, sin embargo, no mostró cambios en la sobrevida con respecto a la de pacientes coronarios que no la recibieron (Enrichd, 2003). Asimismo, otro subestudio del mismo grupo concluyó que aquellos pacientes con depresión refractaria al tratamiento óptimo estándar (terapia cognitivo - conductual y sertralina) tienen mayor mortalidad tardía post IAM con respecto a aquellos que presentan buena respuesta.

La anhedonia por sí sola (Davidson et al., 2010) sería de utilidad para identificar aquellos pacientes con mayor riesgo de eventos cardiovasculares mayores adversos, con un Hazard ratio de 1.58 (Cl 95\% 1.16-2.14; $\mathrm{p}<0,01)$.

Una reciente revisión sistemática de Cochrane (Richards et al., 2017) mostró que no hubo reducción del riesgo de mortalidad total o asociada a procedimientos de revascularización con psicoterapia comparado a la ausencia de la misma. Además, no hubo reducción de IAM no fatales. Sin embargo, se describe un $21 \%$ de reducción en la mortalidad cardiaca global.

La misma revisión mostró una importante heterogeneidad estadística para todos los outcomes psicológicos, no así los clínicos. Las meta-regresiones de ciertas intervenciones psicológicas mostraron no ser significativas en la mortalidad cardiaca y total. En la depresión, las intervenciones combinadas con una terapia farmacológica apropiada aparentemente serían más efectivas que la intervención psicológica por sí sola.

En personas con enfermedad coronaria, no existe evidencia de que los tratamientos psicológicos tuviesen un efecto en la mortalidad total, el riesgo de procedimientos de revascularización o en el rango de IAM no fatal. Sin embargo, la tasa de mortalidad cardiaca fue menor, y los síntomas psiquiátricos (depresión, ansiedad o estrés) fueron aliviados (Richards et al. 2017).

\section{Depresión como factor de riesgo cardiovascular}

Es interesante abordar la depresión como factor de riesgo coronario en la población sana. En el mismo estudio de Blumel abordan la temática, reportando una prevalencia de $6,4 \%$ de IAM en pacientes con depresión. Sin embargo, no fue posible realizar una asociación significativa entre la dicha ocurrencia y la depresión de base. 
Lo anterior también se refleja en la literatura internacional, donde no ha podido establecerse claramente si la depresión es un factor de riesgo para enfermedad coronaria o un agravante en el pronóstico de la misma (Pickering et al., 2004).

Otro subestudio ENRICHD (Bekke-Hansen et al., 2011) mostró que los síntomas somáticos de depresión a los 12 meses post IAM en pacientes con riesgo psicosocial aumentado, evaluados a través del Instrumento de Apoyo Social del ENRICHD (un cuestionario de cinco elementos diseñado para este estudio, que permite percibir el apoyo tanto emocional como funcional), predicen la mortalidad posterior, sugiriendo que las intervenciones psicosociales dirigidas a los síntomas depresivos somáticos podrían mejorar el pronóstico cardiovascular.

Diversos autores (Lane et al., 2003; Pickering, et al., 2004) enfatizan que los ensayos Enrichdy Sadhart correlacionan la depresión como un predictor de mortalidad solo en presencia de enfermedad coronaria, no así en otras condiciones cardiovasculares tales como la Fracción de Eyección del Ventrículo Izquierdo, o la clasificación clínica del IAM al momento de su inicio (Killip). Pese a que el Hazard ratio fue de 2.8 para todas las causas de mortalidad, una vez ajustándolo para diabetes, tabaquismo, disfunción ventricular y otros factores médicos, demográficos y terapéuticos, el HR solo bajo a 2.4, lo que refleja el potencial de ser un factor de riesgo en pacientes con enfermedad coronaria (como lo citado en el estudio Enrichd), lo que apoya su consideración al momento de establecer la prevención secundaria. Asimismo, no existe evidencia suficiente que apoye que la depresión en sujetos sanos tiene efectos en los eventos adversos cardiovasculares.

Otros autores (Kronish et al., 2009) plantean la interrogante sobre si la depresión posterior a un IAM actuaría como un marcador de factores pronósticos conocidos de mortalidad. En este estudio, tanto la severidad de síntomas depresivos auto-reportados a través del cuestionario de depresión de Beck (BDI) como el diagnóstico clínico de depresión mayor, no tuvieron asociación estadísticamente significativa con el score de GRACE. Gran parte de los pacientes con diagnóstico de depresión además eran dislipidémicos y/o diabéticos, a diferencia del grupo control. Sin embargo, este estudio al momento de incorporación no incluyó pacientes con un puntaje en el BDI entre 5 y 9. La mayoría de personas de este grupo parecía no deprimida al momento de la entrevista clínica. Pese a lo anterior, dicha ausencia de rango intermedio no afectaría -según los autores- la falta de asociación entre la depresión y elementos del GRACE.

Un subestudio del grupo SADHART (Glassman et al., 2009) mostró que la severidad de la depresión mayor durante la hospitalización, así como la falla de su mejoría en los 6 meses posteriores a un IAM, predice más del doble de la mortalidad a los 6.7 años de seguimiento. Además, la depresión persistente aumenta la mortalidad y disminuye la adherencia terapéutica, debiendo ser tratada de forma agresiva, siendo diligente en la promoción de la adherencia a la terapia cardiovascular

La asociación entre la depresión tras un IAM y la elevada morbimortalidad cardiaca puede deberse a un aumento de la severidad de la enfermedad cardiaca. El meta análisis de pacientes individuales de A. Meijer concluyó que la asociación entre la depresión post IAM y el pronóstico luego del ajuste para severidad de enfermedad cardiaca se atenuaba, sin embargo, la depresión continuó estando asociada de forma independiente al pronóstico, con un $22 \%$ de riesgo aumentado en todas las causas de mortalidad y un $13 \%$ de riesgo aumentado en eventos cardiovasculares por desviación estándar en el z-score de depresión.

La depresión puede considerarse un factor de riesgo de mortalidad luego de un IAM (Scherrer et al., 2012). Los pacientes con depresión refractaria tienen peor pronóstico versus aquellos que sí responden. Lo anterior recae en múltiples aristas, como el cumplimiento terapéutico y adherencia a cambios de estilo de vida, así como mecanismos moleculares poco dilucidados. Asimismo, se ha visto que la mortalidad por todas las causas post IAM es mayor en los pacientes con depresión mal tratada y es un riesgo en los pacientes con depresión refractaria.

Por otra parte, la depresión post IAM se asocia a mayores ingresos hospitalarios, principalmente por causas cardiovasculares (Myers et al., 2012). Además, existe una adopción reducida de medicinas de prevención secundarias en estos pacientes, siendo importantes en términos de pronóstico y de calidad de vida de los pacientes, así como gastos sanitarios, siendo recomendable un seguimiento cercano de síntomas subclínicos de depresión y su tratamiento temprano.

Un estudio de Zuidersma et al. (2013 a). Demostró con un grupo randomizando de pacientes con depresión post IAM, formando un grupo que recibió tratamiento antidepresivo farmacológico y no farmacológico y otro grupo sin tratamiento por 12 meses que no existían diferencias entre ambos grupos en la reducción de la morbimortalidad cardiovascular, por lo que la implementación de tratamiento antidepresivo en los pacientes con depresión post IAM no reduciría su morbimortalidad.

En otro estudio del mismo grupo Zuidersma et al. (2013 b). Se demostró que los síntomas depresivos reportados por los mismos pacientes son predictores más adecuados de la morbimortalidad 
cardiovascular que la depresión clínica, existiendo una gran confusión con la severidad de la enfermedad cardiovascular. Complementando lo anterior, en otro estudio del mismo autor se demostró que cada síntoma adicional de depresión está significativamente asociado a un 15\% de mayor riesgo de nuevos eventos cardiovasculares, siendo más fuerte en los pacientes no depresivos que en los depresivos, independientemente de la severidad de la enfermedad cardiovascular de base.

\section{Fisiopatología entre la depresión y eventos adversos cardiovasculares}

Existe evidencia que demuestra que los pacientes con depresión previa tienen niveles más elevados del propéptido natriurético cerebral N-terminal (NT-proBNP) y Troponina I ( $\mathrm{Tnl}$ ) con respecto a aquellos sin depresión, siendo solo de significancia estadística el NT-proBNP (Ren et al., 2017). Esta asociación se ha visto en otros ensayos clínicos, como en personas ancianas con síntomas depresivos y diabetes (Feinkohl et al., 2012)

El proBNP y NT-proBNP son sintetizados en los cardiomiocitos en respuesta a estrés parietal, estando conocidamente elevados en situaciones como IAM e insuficiencia cardiaca (James et al., 2003). En un subestudio del grupo GUSTO (Global Utilization of Strategies To Openoccluded arteries) se describió que estos marcadores tienen una estrecha relación con la mortalidad a corto y largo plazo post IAM. Vale la pena mencionar otro estudio en el que se concluyó que la insuficiencia cardiaca en pacientes con depresión mayor presentó mayores niveles de BNP, pero menor disfunción cardiaca que aquellos con depresión moderada o sin depresión (Aguilar et al., 2010).

Tanto la depresión como el IAM generan aumento de la actividad del eje neuroendocrino y del nivel de citoquinas proinflamatorias circulantes. Por lo tanto, es esperable que cuando ambas condiciones se encuentran presentes, los cambios fisiopatológicos de cada trastorno se encuentren potenciados, exacerbando las manifestaciones clínicas de cada uno (Ren et al., 2017).

Otros autores plantean que la depresión atípica y melancólica podría ser concebida como una desregulación del sistema de respuesta al estrés (Gold et al., 2015). La desregulación de la corteza prefrontal y la amígdala genera un aumento de la ansiedad e inhibición del eje Hipotalámico-Hipofisiario-Adrenal (HHA), así como de los sistemas noradrenérgicos del Locus Ceruleus (LC-NE). Lo anterior contribuye al desarrollo de manifestaciones sistémicas tales como enfermedad coronaria prematura y osteoporosis, lo que se ve corregido llevando las funciones prefrontales y amigdalianas a estados no estresados bajo tratamiento con ISRS e inhibidores de la monoaminooxidasa (IMAO).
En estudios post mortem se evidenció hiperplasia de las células productoras del Factor liberador de corticotropinas (CRT) en pacientes con enfermedad coronaria. Los niveles aumentados de corticoides circulantes contribuirían al desarrollo de aterosclerosis, hipercolesterolemia, hipertensión e hipertrigliceridemia, pudiendo explicar en parte la asociación entre trastornos depresivos y enfermedad coronaria (Khawaja et al., 2009).

En el estudio anterior se señala al tono autonómico alterado como responsable de la baja variabilidad cardiaca, lo cual sería en parte responsable de la aparición de arritmias post IAM. Sin embargo, lo anterior todavía carece de evidencia fuerte, quedando interrogantes sobre la fisiopatología detrás de la alteración de la actividad de los barorreceptores (hipoactividad) y la variabilidad del intervalo QT.

La variabilidad cardiaca reducida, sensibilidad disminuida de los barorreceptores, aumento de la presión de pulso y aumento de los niveles plasmáticos de Proteína C Reactiva de alta sensibilidad (hsCRP) están asociados con un aumento del riesgo de eventos cardiacos futuros tanto en sujetos sanos como en cardiópatas coronarios conocidos (Dawood et al., 2007). En este ensayo, en el grupo de pacientes en tratamiento con inhibidores selectivos de la recaptación de serotonina (ISRS), se encontró disminución de la actividad de barorreceptores, variabilidad cardiaca reducida, presión de pulso aumentada y niveles plasmáticos de hsCRP con respecto a pacientes sanos, lo cual podría tener implicancias en el mayor riesgo de muerte cardiovascular, destacando que existe una gran mejoría en los síntomas depresivos y ansiosos por sí solos utilizando este medicamento, pero no pudiendo descartar su rol en el desarrollo de los fenómenos anteriormente mencionados.

Con respecto a la activación de la respuesta inflamatoria sistémica, el aumento de citoquinas proinflamatorias circulantes podría llegar a mecanismos de neuroinflamación central (Liu et al., 2013), la que puede seguir en el tiempo independientemente de la disminución de la inflamación periférica. Un potencial mecanismo en la relación entre la depresión y el estado post IAM podría ser el Factor de Necrosis Tumoral Alfa, pudiendo ser efectivo como blanco terapéutico hacia la antiinflamación, disminuyendo la tasa de depresión y pudiendo mejorar los outcomes cardiovasculares. No obstante, lo anterior aún continúa en discusión y carece de fuerza estadística suficiente para poder ser una opción terapéutica en la actualidad.

Otros mecanismos fisiopatológicos descritos en la literatura, como la asociación entre la depresión y un aumento de la ectopia ventricular y agregación plaquetaria, tiene poca fuerza científica en la actualidad, y no han podido ser objetivadas del todo (Glassman, 2008). 


\section{Antidepresivos y eventos cardiovasculares}

En contraste con el estudio de Dawood, una revisión sistemática que comparó tratamientos farmacológicos convencionales con el placebo en el desarrollo de eventos cardiacos adversos (Swenson et al., 2006) concluyó que comparando tres grupos de tratamientos farmacológicos para la depresión (ISRS, antidepresivos tricíclicos, otras terapias activas) con placebo en pacientes cardiópatas y/o antecedente de accidente cerebrovascular, no se detectaron diferencias significativas en los Odds ratio para eventos cardiovasculares adversos serios (muerte debido a falla cardiaca, ACV e IAM) y no serios (palpitaciones, dolor torácico, angor, arritmias, hipertensión, síncope y eventos no específicos neurológicos-cardiovasculares).

Otra revisión sistemática comparó los posibles efectos cardiovasculares de los fármacos más comúnmente utilizados en el tratamiento de la depresión (Marano et al., 2009), lo que se expone en la Tabla 1.

Tabla 1: Impacto cardiovascular de los fármacos más comúnmente utilizados en el tratamiento de la depresión. Adaptado de Marano et al. (2009).

\begin{tabular}{|c|c|}
\hline Fármaco & Impacto cardiovascular \\
\hline Fluoxetina & $\begin{array}{l}\text { Aparentemente segura en muestras pequeñas de } \\
\text { pacientes ancianos con depresión y enfermedad } \\
\text { cardiaca. La inhibición selectiva de recaptación } \\
\text { de serotonina no causó hipotensión ortostática o } \\
\text { disminución de la conducción, así como no tuvo } \\
\text { actividad antiarrítmica. En pacientes post IAM no } \\
\text { hubo disminución en la función cardiaca estudiada } \\
\text { en el ecocardiograma ni electrocardiograma. }\end{array}$ \\
\hline Venlafaxina & $\begin{array}{l}\text { Se ha visto un aumento en la presión sistólica y } \\
\text { diastólica con dosis mayores a } 300 \mathrm{mg} / \text { día. No } \\
\text { existen estudios sistemáticos sobre su uso, por lo } \\
\text { que no es posible considerarlas seguras en pacientes } \\
\text { cardiópatas con depresión concomitante. }\end{array}$ \\
\hline Sertralina & $\begin{array}{l}\text { No afectó la frecuencia cardiaca ni la presión arterial } \\
\text { sistólica de pie. Además, no tuvo efecto significativo } \\
\text { en la variabilidad cardiaca, incluyendo la total y de } \\
\text { alta frecuencia. Además, tendría efectos endotelio- } \\
\text { protectores y antiplaquetarias. El tratamiento no se } \\
\text { asoció a cambios en la función cardiaca, frecuencia } \\
\text { cardiaca o arritmias con respecto al placebo. }\end{array}$ \\
\hline Mirtazapina & $\begin{array}{l}\text { Su riesgo radica en la ganancia significativa de } \\
\text { peso, siendo ello un factor de riesgo cardiovascular } \\
\text { independiente }\end{array}$ \\
\hline Citalopram & $\begin{array}{l}\text { Fue superior al placebo reduciendo el HAM-D score } \\
\text { a las } 12 \text { semanas. No existe diferencia significativa } \\
\text { con el placebo con respecto a la modificación de } \\
\text { la presión arterial o cambios electrocardiográficos. } \\
\text { A las } 12 \text { semanas hubo un gran aumento en el } \\
\text { Óxido Nítrico Total (tNO) respecto al placebo. No } \\
\text { hubo diferencia con respecto a otros marcadores } \\
\text { (P-selectina, B-Trombomodulina e ICAM-1 soluble). }\end{array}$ \\
\hline
\end{tabular}

\section{Ansiedad post IAM}

La ansiedad se asocia directamente con peor calidad de vida en los 10 años posterior a un IAM, orientando a la necesidad del enfoque tanto a las reacciones ansiosas como depresivas al momento temprano y tardío del diagnóstico de un IAM (Benyamini et al., 2013). Muchas de estas asociaciones son explicadas por mecanismos análogos a los de la depresión, debiendo tener especial énfasis en el viraje hacia el desarrollo de depresión.

La misma revisión de Cochrane antes descrita (Richards et al., 2017) mostró que las intervenciones realizadas en sujetos ansiosos con una condición psicológica conocida previa mostraron ser más efectivas que aquellas indicadas en población no seleccionada.

\section{Delirium post IAM}

El delirium se define como un trastorno agudo de la atención y cognición visto de manera relativamente frecuente en personas sobre 65 años o más. La prevalencia se estima entre 11 y $42 \%$ en hogares de ancianos, así como en hospitalizaciones domiciliarias y unidades de larga estadía (Kukreja et al. 2015).

Con respecto a la relación entre el delirium y el IAM, esta se da principalmente al momento de admisión de los pacientes susceptibles (edad avanzada, alto número de medicamentos, enfermedad física de importante repercusión sistémica, alteraciones metabólicas, sepsis, etc.) a las unidades coronarias, el cual sigue un comportamiento similar a otras patologías de unidades de paciente crítico (Lahariya et al., 2013). De aquellos pacientes que ingresan a Unidades Coronarias, un cuarto desarrolla delirium, y de ellos, un $27 \%$ murió durante su hospitalización, además de tener mayores estadías en UPC.

El mismo estudio destaca que muchos de los aspectos gatillantes del delirium en este grupo de pacientes son modificables, sugiriendo la evaluación dirigida a los pacientes que ingresen o permanezcan en estas unidades, para revertir las causas modificables que puedan llevar al desarrollo de este trastorno.

\section{Trastorno de personalidad D post IAM}

Los pacientes con personalidad tipo $D$ son aquellos que tienen tendencia a experimentar emociones negativas y ser socialmente inhibidos. En el estudio de Williams et al. (2013), concluyeron que este grupo de pacientes tienen un perfil distintivo hacia su enfermedad, lo cual podría explicar los mayores efectos adversos vistos en ellos luego de un IAM. Otro estudio demostró en un grupo de pacientes cardiovasculares que aquellos de sexo femenino con personalidad tipo D, Desesperanzados, Ansiosos y Depresivos 
tuvieron mayores puntuaciones de riesgo cardiovascular y menor autoestima, sugiriendo especial atención para este grupo de pacientes en dicho contexto (Montero et al., 2012).

\section{Estrés post IAM}

Arnold et al. (2012) demostraron que la percepción de estrés moderado y alto al momento de un IAM está asociado con peores outcomes a largo plazo. No existen estudios que determinen claramente la prevalencia de esta patología como fenómeno paralelo post IAM, por lo que los mismos autores recomiendan la realización de dichos estudios complementarios.

En pacientes con diagnóstico reciente de IAM o angina estable, la presencia de estrés mental puede lograr inducir fenómenos isquémicos, aumentando la mortalidad global respecto a quienes no lo padecen, según lo descrito desde el estudio PIMI (Psychophysiological Investigations of Myocardial Ischemia Study). Esto puede ser explicado a través de tres diferentes posibles mecanismos:

1. Aumento de la presión arterial sistólica a niveles similares a los alcanzados durante el ejercicio.

2. Altas respuestas a la adrenalina y otras catecolaminas, gatillando que el cambio en la presión arterial sea más rápido que en el ejercicio, junto con un aumento en la resistencia vascular coronaria, favoreciendo fenómenos de isquemia.

3. Posible reducción en el aporte de oxígeno miocárdico, posiblemente por afección de la microcirculación, además del fenómeno descrito en el punto 2.

Parte de la fisiopatología que involucra los efectos deletéreos del estrés en la morbimortalidad cardiovascular fueron revisados en el subtema correspondiente dentro del análisis de la depresión e IAM.

\section{Discusión}

La cardiopatía isquémica en la actualidad es considerada un buen modelo de enfermedad biopsicosocial (Bancalero et al., 2013). En base a las diferentes aristas de la literatura, la Depresión Mayor no tratada es considerada un factor de riesgo cardiovascular independiente en pacientes con enfermedad coronaria, requiriendo ser considerada dentro del proceso de rehabilitación cardiaca, con medidas activas para su tratamiento.

Los mecanismos fisiopatológicos responsables de la asociación entre depresión, ansiedad y otras enfermedades psiquiátricas con el aumento de la morbimortalidad cardiovascular post IAM aún son tema de estudio y discusión. En base a lo anterior, también se ha observado una variación significativa y positiva en dicha morbimortalidad en este grupo de pacientes sometidos a diferentes intervenciones en sus conductas, estilos de vida y diversos factores biopsicosociales (Cabrera et al., 2011). Además, se ha visto que los cambios en el apoyo social durante la fase temprana de un IAM son importantes como predictores de outcomes, pudiendo significar mejorías a largo plazo si se interviene tempranamente.

Existen diversos estudios que buscan aplicar escalas de apoyo social en pacientes cardiovasculares, que podrían tener efectos positivos en la prevención de eventos asociados a la morbimortalidad en este grupo (Leifheit-Limson et al., 2012). En Chile, el estudio de Poblete et al. (2015) demostró la aplicabilidad en grupos focales y entrevistas semiestructuradas utilizando la escala del Medical Outcomes Study Social Support Survey (MOS-SSS) para medir el concepto de "apoyo social", demostrando que el instrumento final tuvo buenas propiedades sicométricas y es aplicable en nuestra población. La evidencia mostrada en esta revisión sugiere la necesidad de la elaboración de estudios prospectivos que reflejen de mejor manera la situación regional, que integren el tratamiento de la depresión en pacientes post IAM, integrándola de manera activa como un marcador independiente de mal pronóstico cardiovascular, debiendo intervenir de manera precoz su aparición para mejorar la calidad de vida y sobrevivencia de estos pacientes.

De la misma manera, se sugiere la realización de estudios que puedan medir la practicidad de diferentes momentos del proceso de enfermedad cardiovascular para intervenir en lo psiquiátrico (intrahospitalario, alta temprana, etc.), así como una cuantificación activa del beneficio de dichas intervenciones, escalas diagnósticas, entre otras estrategias antes citadas.

\section{Referencias}

Aguilar VB, Ochiai ME, Cardoso JN, Del Carlo CH, Morgado PC, Munhoz RT (2010). Relationship between depression, BNP levels and ventricular impairment in heart failure. Arquivos Brasileiros de Cardiología 95, 732-737.

Alhurani A, Lennie T, Moser D (2015). Predisposing Factors of Persistent Symptoms of Depression and Anxiety Among Patients With Acute Coronary Syndrome. Circulation 132, a18551

Arnold SV, Smolderen KG, Buchanan DM, Li Y, Spertus JA (2012). Perceived Stress in Myocardial Infarction. Journal of the American College of Cardiology 60, 1756-1763

Bancalero C, Aguilera A, Navarro M, Carrión L, Quirós A, Ruiz S (2013). Fundamentos teóricos de la intervención psiquiátrica y psicológica de enlace en unidades de rehabilitación cardiaca. $C$. Med. Psicosom 105, 20-30 
BenyaminiY, Roziner I, Goldbourt U, DroryY, GerberY (2013). Israel Study Group on First Acute Myocardial Infaction. Depression and Anxiety Following Myocardial Infarction and Their Inverse Associations with Future Health Behaviors and Quality of Life. Ann. Behav.Med. 46, 310-321

Berkman L, Blumenthal J, Burh M, Carney R, Catellier D, Czajkowski S, De-Busk R, Hosking J, Jaffe A, Mitches P, Norman J, Powell L, Schneiderman N, Gables C (2003). Effects of Treating Depression and Low Perceived Social Support on Clinical Events After Myocardial Infarction. The Enhancing Recovery in Coronary Heart Disease Patients (ENRICHD) Randomized Trial. JAMA 289, 3106-3116

Blumel B, Gibbons A, Kanacri A, Kerrigan N, Florenzano R (2005). Depressive symptoms after an acute myocardial infarction. Revista Médica de Chile 133, 1021-1027.

Cabrera J, Gandarilla JC, González I (2011). Nuevos aspectos fisiopatológicos en la intervención psicosocial de las personas con enfermedad arterial coronaria. Revista Cubana de Medicina. 50, 83-93

Calderón J, Gabrielli L, Gonzalez M, Villarroel L, Castro P, Corbalán R (2010). Depressive Symproms Among Patients with acute myocardial infarction. Relationship with revascularization therapy. Revista Médica de Chile 138, 710-706.

Carney R, Blumenthal J, Freedland K, Youngblood M, Veith R, Burg M, Cornell C, Saab P, Kaufman P, Czajkowski S, Jaffe A (2004). Depression and Late Mortality After Myocardial Infarction in the Enhancing Recovery in Coronary Heart Disease (ENRICHD) Study. Psychosomatic Medicine 66, 466-474

Davidson K, Burg M, Kronish I, Shimbo D, Dettenborn L, Mehran R, Vorchheimer D, Clemow L, Schwartz J, Lesperance F, Rieckman $\mathrm{N}$ (2010). Association of Anhedonia With Recurrent Major Adverse Cardiac Events and Mortality 1 Year After Acute Coronary Syndrome. Archives of General Psychiatry 67, 480-488

Dawood T, Lambert E, Barton D, Laude D, Elghozi JL, Esler M, Haikerwal D, Kaye D, Hotchkin E, Lambert G (2007). Specific Serotonin Reuptake Inhibition in Major Depressive Disorder Adversely Affects Novel Markers of Cardiac Risk. Hypertension Research 4, 285-293

Deepti Kukreja, Ulf Gunther, Julius Popp (2015). Delirium in the elderly: current problems with increasing geriatric age. Indian Journal of Medical Research 142, 655-662.

Feinkohl I, Sattar N, Welsh P, Reynolds RM, Deary IJ, Strachan MW (2017). Association of Nterminal probrain natriuretic peptide with cognitive function and depression in elderly people with type 2 diabetes. Chinese Medical Journal 130, 542-548.
Glassman A, BiggerT, Gaffney M (2009). Psychiatric Characteristics Associated With Long-term Mortality Among 361 Patients Having an Acute Coronary Syndrome and Major Depression: Seven-Year Follow-up of SADHART Participants. Archives of General Psychiatry 66, $1022-1029$.

Glassman A (2007). Depression and cardiovascular comorbidity. Dialogues in Clinical Neuroscience 9, 9-17.

Gold P, Machado-Vieira R, Pavlatou M (2015). Clinical and Biochemical Manifestations of Depression: Relation to the Neurobiology of Stress. Neural Plasticity 2015

James SK, Lindahl B, Siegbahn A, Stridsberg M, Venge P, Armstrong $P$ (2003). N-terminal probrain natriuretic peptide and other risk markers for the separate prediction of mortality and subsequent myocardial infarction in patients with unstable coronary artery disease: A Global Utilization of Strategies To Open occluded arteries (GUSTO) IV substudy. Circulation 108, 275-281

Khawaja I, Westermeyer J, Gajwani P, Feinstein R (2009). Depression and coronary artery disease: The association, mechanisms, and therapeutic implications. Psichiatry 6 p38-51.

Kronish I, Rieckmann N, Schwartz J, Schwartz D, Davidson K (2009). Is Depression After an Acute Coronary Syndrome Simply a Marker of Known Prognostic Factors for Mortality?. Psichosomatic Medicine 71, 697-703.

Kukreja D, Gunther U, Popp J (2015). Delirium in the elderly: current problems with increasing geriatric age. Indian Journal of Medical Research 142, 655-662

Lahariya S, Grover S, Bagga S, Sharma A (2014). Delirium in patients admitted to a cardiac intensive care unit with cardiac emergencies in a developing country; incidence, prevalence, risk factor and outcome. General Hospital Psychiatry 36, 156-164

Lanas F, Potthoff S, Mercadal E, Santibáñez C, Lanas A, Standen D (2008). Individual and population risk in acute myocardial infarction: The Chilean INTERHEART study. Rev. med. Chile. 136, 555-560

Leifheit-Limson EC, Reid KJ, Kasi SV, Lin H, Bunchanan DM, Jones PG, Peterson PN, Parashar S, Spertus JA, Lichtman JH (2012). Changes in social support within the early recovery period and outcomes after acute myocardial infarction. Journal of Psychosomatic Research 73, 35-41.

Liu H, Luiten PG, Eisel UL, Dejongste MJ, Schoemaker RG (2013). Depression after myocardial infarction:TNF-a-induced alterations of the blood-brain barrier and its putative therapeutic implications. Neuroscience and Biobehavioral Reviews 37, 561-572 
Marano G, Harnic D, Lotrionte M, Biondi-Zoccai G, Abbate A, Romagnoli E, Mazza M (2009). Depression and the cardiovascular system: increasing evidence of a link and therapeutic implications. Expert Review of Cardiovascular Therapy 7, 1123-1147

Montero P, Rueda B, Bermudez J (2012). Relación de la personalidad tipo $D$ y el agotamiento vital con las emociones negativas y el ajuste psicológico a la enfermedad cardiaca. Revista de Psicopatología y Psicología Clínica 17, 93-106

Myers V, Gerber Y, Benyamini Y, Goldbourt U, Drory Y (2012). Post-myocardial infarction depression: Increased hospital admissions and reduced adoption of secondary prevention measures - A longitudinal study. Journal of Psychosomatic Research 72, 5-10

Organización Mundial de la Salud (2015). "Enfermedades cardiovasculares - nota de prensa". Disponible en http://www.who.int/mediacentre/factsheets/fs317/es/.

Pickering T, Davidson K, Shimbo D (2004). Depression as a Risk Factor for Post-MI Mortality. Journal Of The American College of Cardiology 44, 469-474

Poblete F, Glasinovic A, Sapag J, Barticevic N, Arenas A, Padilla O (2015). Apoyo social y salud cardiovascular: adaptación de una escala de apoyo social en pacientes hipertensos y diabéticos en la atención primaria chilena. Atención Primaria 47, 523-531.

Reed G, Rossi J, Cannon C (2017). Acute Myocardial infarction. The Lancet 389, 197-210

Ren Y, Jia J, Sa J, Oiu L, Cui Y, Zhang Y, Yang H, Liu G (2017). Association between N-terminal proB-type Natriuretic Peptide and Depressive Symptoms in Patients with Acute Myocardial Infarction. Chinese Medical Journal 130, 542-8
Richards SH, Anderson L, Jenkinson CE, Whalley B, Rees K, Davies $\mathrm{P}_{\text {, }}$ Bennett P, Liu Z, West R, Thompson DR, Taylor RS (2017). Psychological interventions for coronary heart disease. Cochrane Database of Systematic Reviews $\mathbf{4}$

Roest, AM, Carney RM, Freedland KE, Martens EJ, Denollet J, de Jonge P (2013). Changes in Cognitive versus Somatic Symptoms of Depression and Event-Free Survival Following Acute Myocardial Infarction in the Enhancing Recovery In Coronary Heart Disease (ENRICHD) Study. Journal of affective disorders 149, 335-341

Sheps D, McMahon R, Becker L, Carney R, Freedland K, Cohen J, Sheffield D, Goldberg D, Ketterer M, Pepine C, Raczynski J, Light K, Krantz D, Stone P, Knatterud G, Kaufmann P (2002). Mental Stress-Induced Ischemia and All-Cause Mortality in Patients With Coronary Artery Disease: Results From the Psychophysiological Investigations of Myocardial Ischemia Study. Circulation 105, 1780-84.

Swenson JR, Doucette S, Fergusson D (2006). Adverse cardiovascular events in antidepressant trials involving high-risk patients: a systematic review of randomized trials. The Canadian Journal of Psychiatry 51, 923-29

Williams L, O'Connor RC, Grubb NR, O'Carroll RE (2011). Type D personality and illness perceptions in myocardial infarction patients. Journal of Psychosomatic Research 70, 141-144

Zuidersma M, Conradi HJ, van Melle JP, Ormel J, de Jonge P (2013 a) Depression treatment after myocardial infarction and long-term risk of subsequent cardiovascular events and mortality: A randomized controlled trial. Journal of Psychosomatic Research 74, 25-30

Zuidersma M, Conradi HJ, van Melle JP, Ormel J, de Jonge P (2013 b). Self-reported depressive symptoms, diagnosed clinical depression and cardiac morbidity and mortality after myocardial infarction. International Journal of Cardiology 167, 2775-2780 